\title{
El papel de la comunicación pública de la ciencia sobre la cultura científica: acercamientos a su evaluación
}

\author{
María del Carmen Sánchez Mora \\ Dirección General de Divulgación de la Ciencia, Universidad Nacional Autónoma de México. Ciudad de \\ México.México.masanche@dgdc.unam.mx \\ ORCID: https:// orcid.org/0000-0002-1877-8502 \\ Alba Patricia Macías Nestor \\ Dirección General de Divulgación de la Ciencia, Universidad Nacional Autónoma de México. Ciudad de \\ México.México.especializacion@dgdc.unam.mx \\ ORCID: https:// orcid.org/0000-0001-8649-6550
}

[Recibido: 23 Febrero 2018. Revisado: 18 Abril 2018. Aceptado: 16 Julio 2018]

\begin{abstract}
Resumen: En la sociedad actual se espera en sus miembros una cultura científica, necesaria para la acción ciudadana y democrática. Se han realizado diversos intentos para evaluar esta cultura por medio de cuestionarios de conocimientos y encuestas masivas de percepción pública de la ciencia y la tecnología, pero escasamente se han considerado en estos registros los efectos de las actividades de comunicación pública de la ciencia. En este trabajo, se propone un modelo para registrar tales efectos en sus distintas modalidades y a través de diferentes medios de comunicación, que van desde el mero entretenimiento hasta el aprendizaje informal y de acuerdo con el tipo de público en el que se pretende incidir, desde masivo hasta individual.
\end{abstract}

Palabras clave: Evaluación; Cultura científica; Comunicación pública de la ciencia; Medios.

The role of public science communication on scientific culture: approaches to its evaluation

Abstract: In today's society its members are expected to acquire a scientific culture necessary for citizen and democratic action. Several attempts have been performed to evaluate this culture by means of questionnaires about knowledge and massive surveys of public perception of science and technology, but the effect of mass media and the activities for public communication of science in the generation of that scientific culture have been scarcely considered in those records. In this work, a model is proposed to register the various effects of public communication of science in its diverse modalities and throught different mass media, ranging from entertainment to informal learning, and according to the type of audience intended to be influenced, from massive to individual.

Keywords: Evaluation; Scientific culture; Public science communication; Media.

Para citar este artículo: Sánchez-Mora C., Macías-Nestor A. (2019) El papel de la comunicación pública de la ciencia en la cultura científica: acercamientos a su evaluación. Revista Eureka sobre Enseñanza y Divulgación de las Ciencias 16(1), 1103. doi: 10.25267/Rev_Eureka_ensen_divulg_cienc.2019.v16.i1.1103

\section{Introducción}

Hoy en día se sabe que en materia de salud, alimentación, vivienda, transporte, comunicaciones, trabajo, tiempo libre, economía y ambiente, las actividades científicas y tecnológicas marcan de manera definitiva a la sociedad. Pese a ello, cuando esta se enfrenta a problemas o situaciones relacionadas con los aspectos anteriores, la ciencia escasamente se ve reflejada en el actuar individual y en la cultura ciudadana. Por ello es necesaria la construcción de una cultura científica a partir de un adecuado proceso educativo realizado a la par de diversos esfuerzos de comunicación, indispensables, entre otras cosas, para la existencia de una democracia verdaderamente participativa en la que la cultura científica funcione como auténtica mediación social entre los ciudadanos, las instituciones y las actividades relacionadas con la ciencia. 
Aun con el ya antiguo reconocimiento de la importancia de que la sociedad deba poseer una cultura científica, de la que se supone debieran formar parte algunos conocimientos básicos, junto con la comprensión amplia y general de los métodos de la ciencia (Durant, Evans y Thomas 1995), por lo regular las definiciones de cultura científica se han enfocado ante todo a describir la posesión de estos y otros contenidos específicos, pero escasamente mencionan las estrategias para su construcción. Es por esta razón que la mayoría de las herramientas para detectarla se basan en el registro de conocimientos individuales de información científica atribuibles comúnmente a la formación educativa formal. Desde luego que la componente escolar de la cultura científica constituye una plataforma indispensable para su construcción, pero poseer una cultura científica implica también, entre otras cosas, poder construir ideas científicas y argumentos racionales, analizar e interpretar evidencias, participar en las estructuras sociales que guían la empresa científica, así como involucrarse en diversas prácticas, no necesariamente escolares, que buscan la alfabetización en ciencia (Brown, Reveles y Gregory 2005). Además, es indispensable considerar la cultura científica como una componente esencial de toda sociedad democrática basada en una economía relacionada con la tecnología y promotora de los valores culturales de la sociedad (Miller 2001). En este sentido reviste particular importancia la llamada cultura científica cívica (Falk y Dierking 2012), que es la posibilidad de que los ciudadanos se mantengan informados sobre los aspectos corrientes de la ciencia, los cuales a su vez les permiten participar activamente en una sociedad involucrada con la ciencia y la tecnología.

Por su parte, para Zamarrón (2006) la cultura científica de la sociedad tiene más que ver con la manera en que los individuos se relacionan con la actividad científica. Para esta autora, si bien una persona con cultura científica requiere contar con información, también necesita una preparación y el desarrollo de habilidades que le permitan situar el conocimiento en su esencia y su sentido, lo que significa poseer una capacidad de análisis y contextualización de lo que sucede en el mundo de la ciencia. Es decir, la cultura científica va más allá de la alfabetización en ciencia, porque si bien los datos, conceptos, teorías, inventos, etc., forman parte de la información mínima de un ciudadano educado, el concepto de cultura científica trasciende el acopio de información que podamos adquirir, y más que el conocimiento, implica la comprensión de la ciencia como producción intelectual y social (Zamarrón 2006).

En este mismo sentido, Burns, O'Connor y Stocklmayer (2003) plantean que la cultura científica comprende al sistema integrado de valores sociales que aprecia y promueve la ciencia per se, así como al conjunto de valores, ethos, prácticas, métodos y actitudes basadas en el universalismo, el pensamiento lógico, el escepticismo organizado y la provisionalidad de los resultados empíricos que existen dentro de la academia o comunidad científica. Nuevamente, no se trata solo del aprendizaje de un vocabulario científico y de su definición estricta, sino de la comprensión y el entendimiento de su significado real, cuando se confronta al ciudadano común con la aplicabilidad efectiva de los términos que este vocabulario contiene. Lo anterior se refiere a un posicionamiento del conocimiento científico en el día a día (Jenkins 1994) para permitir la participación activa de los ciudadanos dentro de la actual sociedad avanzada en cuestiones de ciencia y tecnología (Falk y Dierking 2012).

Hasta aquí puede verse que de estas y muchas otras concepciones existentes sobre la cultura científica emergen dos perspectivas principales; primero, la que valora los conocimientos científicos, las prácticas, los hábitos mentales y las formas de usar el conocimiento por los ciudadanos para adquirir nuevos conocimientos científicos (perspectiva criticada porque pareciera ser abstracta y sobre todo, desconectada de la experiencia); y una segunda perspectiva, llamada sociocultural, que busca situar a la cultura científica en la vida diaria, de manera que la ciencia sea manejable en un contexto social relevante. Sin embargo, estas dos visiones no se contradicen, en tanto una propone la adquisición de conocimiento como 
preparación para la participación social, mientras que la segunda plantea el involucramiento en actividades sociales que empleen el conocimiento científico y tecnológico (Brown et al. 2005).

Si se revisan las posturas anteriormente citadas, es posible ver que, sin embargo, no consideran el proceso comunicativo, indispensable para que la ciencia se integre a la cultura, pues a través de él se transmiten no solo los conceptos científicos básicos, sino también las controversias entre expertos, o la construcción social del conocimiento, que incluye los resultados y valores científicos, el conocimiento popular y la percepción social de las ciencias (Vaccarezza 2008).

Para distinguirla de la comunicación de la ciencia entre pares, la Comunicación Pública de la Ciencia (CPC) se define como un campo de conocimiento multi, inter y transdisciplinario, que conjunta saberes provenientes de diversas áreas, tales como las ciencias naturales, exactas, de la salud, tecnologías, ingenierías y, recientemente, sociales y humanísticas, así como el manejo de los distintos medios de comunicación y el conocimiento de los diferentes públicos (fundamentos de la DGDC, UNAM).

Para que este proceso comunicativo se lleve a cabo, los diferentes medios para la CPC adquieren un papel preponderante y, por tanto, su eficacia requiere ser evaluada. Este asunto constituye una enorme y complicada empresa debido a la gran cantidad de medios que se emplean para la comunicación, la diversidad de actores que intervienen y, desde luego, la variedad de servicios que los medios de CPC prestan a la sociedad, que van desde dar apoyo al sistema escolarizado hasta simplemente llenar el tiempo libre. Pero, sobre todo, la evaluación de las acciones llevadas a cabo por estos medios está estrechamente ligada a los propósitos perseguidos por los diferentes esfuerzos comunicativos, que pueden ser tan amplios como entretener, enseñar en diversos grados, buscar la comprensión de ciertos temas científicos, propiciar el acercamiento a las ciencias, promover la motivación por la ciencia, generar vocaciones, etc. (Reynoso 2001).

\section{La influencia de la CPC sobre la cultura científica como punto de partida}

Actualmente se han multiplicado las instituciones que realizan actividades de CPC las cuales tienen variados objetivos, entre los cuales destacan divertir, informar, capacitar, educar, sensibilizar, empoderar o generar la apropiación social de la ciencia y la tecnología.

La proliferación de estas actividades pone en tela de juicio el impacto social de la CPC, al que va aparejado la dificultad para evaluarla, particularmente cuando se considera que su meta es la búsqueda de una cultura científica en la población, aspecto difícil de medir cuando no se ha partido de una definición funcional de cultura científica. A la dificultad anterior habrá que sumar que existe una fuerte discusión acerca de si los resultados de la CPC se deben medir en los individuos o en las comunidades (Schauble, Leinhart y Martin 1997).

Hasta aquí se ha mencionado que la formación de una cultura científica implica tanto un proceso educativo (no necesariamente formal) como uno de comunicación de la ciencia, el cual, debido a su complejidad, dificulta la evaluación tanto del propio proceso comunicativo como de sus resultados. Por lo anterior y para efectos comparativos de la eficacia que diferentes acciones en pro de la CPC pudieran tener en la generación de la cultura científica de una sociedad, se hace necesario desglosar de inicio los diferentes medios intervinientes y sus efectos sobre los diferentes receptores, de manera que se puedan hacer comparaciones de los resultados, por lo menos en países con problemáticas sociales y educativas semejantes como es el caso de los latinoamericanos. En esta región política, difícilmente se pueden aplicar evaluaciones que parten de la consideración de que los ciudadanos cuentan con una adecuada plataforma de conocimientos producto de la enseñanza formal, cuando la realidad es que el 
acercamiento a la ciencia proviene fundamentalmente de otro tipo de esfuerzos, como los realizados por los medios para la CPC.

Solo con el punto de partida de una plataforma común, apoyada en el lenguaje y los métodos propios de la comunicación de la ciencia, se podrán desarrollar indicadores que ayuden a valorar el desempeño de la CPC en términos de alcance y eficiencia, y sentar así las bases para poder hacer comparaciones al interior de cada proyecto de comunicación, $y$, más adelante, a partir de indicadores semejantes desarrollados en otras instituciones y países, lograr aproximarse a las mejores prácticas en términos de comunicación. De esa manera se puede aprovechar la experiencia, los éxitos y los fracasos que ya han ocurrido en otros medios. Esta sería una primera etapa a abordar antes de incursionar en el desarrollo de mediciones sobre el amplio concepto de la cultura científica en contextos particulares.

Por ello es que el reto más apremiante por ahora es el de la evaluación del impacto de las iniciativas de comunicación de la ciencia en el público receptor, que a su vez permitirá considerar el cumplimiento de objetivos de cualquier proyecto de CPC. Queda pendiente, desde luego, la consideración sobre la cultura científica que puede tener la escuela formal, así como la introducción explícita de la construcción social de la ciencia en las acepciones señaladas anteriormente.

\section{Los efectos de la CPC a partir del reconocimiento del aprendizaje informal}

De todos los medios de CPC, han sido los museos de ciencia los que probablemente más han aportado al conocimiento de su proceso, ya que al tener acceso directo a sus usuarios, han permitido reconocer que el receptor (visitante en este caso) construye su propio conocimiento y comprensión de la ciencia de acuerdo a sus intereses y motivaciones (Gregory y Miller 1998). Hoy se sabe que dicha construcción es de naturaleza personal y muy diferente del fenómeno que ocurre dentro de los espacios educativos formales, por lo que se le ha denominado aprendizaje informal (Rennie 2001).

A partir del reconocimiento de esta forma de aprendizaje, se pudo describir un fenómeno educativo que por muchos años se estaba tratando de detectar como resultado de la interacción con los medios de CPC, y que, dadas sus características tan diferentes al proceso educativo formal ya no podía seguirse evaluando con los métodos tradicionales pues se llegaba a resultados poco objetivos. La aceptación de esta modalidad educativa implica una visión totalmente diferente de lo que por mucho tiempo se consideró aprendizaje. Percibirlo como un proceso holístico ha implicado una gran dificultad para documentarlo (Rennie y McClafferty 1996), y más aún, comprender el resultado de la CPC como un tipo de aprendizaje de corte informal requirió considerar el fenómeno de aprendizaje ya no como un resultado, sino como un proceso, cuyo registro requiere de nuevos enfoques metodológicos. Cabe mencionar que el reconocimiento del fenómeno de aprendizaje informal no es reciente: de alguna manera había empezado a ser descrito por varios autores desde hace un par de décadas (Borun, Massey y Lutter 1993).

La conciencia del proceso de aprendizaje informal ha permitido también el desarrollo de instrumentos para su detección, muchos de ellos traducidos en categorías de acciones, habilidades y actitudes codificables, que incluso han servido a muchos medios (en particular a los museos) como índices de efectividad de estos medios, y que permiten justificar la inversión gubernamental o privada que en ellos se hace. Entre las aportaciones más interesantes al respecto pueden señalarse los Generic Learning Outcomes (GLO) desarrollados por el equipo de trabajo de E. Hooper Green-Hill (2008) y los Strands de la National Academy of Sciences 
de EUA (National Research Council 2009). Estos últimos, como valiosos esfuerzos para generar rúbricas que evalúan la eficacia y el alcance de la modalidad educativa informal.

Sin duda las investigaciones más recientes sobre el aprendizaje informal reconocen que se trata de un evento fugaz y al mismo tiempo acumulativo, totalmente dependiente de un gran número de variables y que se ve influido por experiencias previas o posteriores vividas por el público, de manera que es difícil encontrar el resultado de un evento que, en sí mismo, representa una mínima parte de un complejo entramado del aprendizaje informal (Diamond, Duke y Utall 2009). Pero es un hecho que este proceso tiene lugar como efecto de los numerosos esfuerzos que hoy en día se realizan fuera del ámbito escolar para acercar la ciencia y la tecnología a las personas alejadas de estas, y que como se verá más tarde, gracias al reconocimiento del impacto de tales iniciativas es posible detectar algunos resultados de ciertas modalidades de la CPC.

La propuesta de evaluación de las actividades para la CPC que más adelante se desarrolla considera el registro del aprendizaje informal de las ciencias como una herramienta metodológica para detectar el efecto de la CPC a partir de ciertos medios que tienen a su interlocutor a la mano o cara a cara, como es el caso de los talleres o las visitas guiadas a los museos, razón por la cual en este apartado se trató el tema del aprendizaje informal.

\section{El registro de la influencia de la CPC sobre la cultura científica}

Existen en la literatura especializada (Godin y Gringas 2000, Bauer et al. 2000, Lewenstein 2003, Burns et al. 2003) varios modelos sobre la comunicación de la ciencia y sus efectos, que buscan comprender la forma en que individuos y sociedad se apropian de la ciencia y la tecnología, que además suelen distinguir entre la cultura de los individuos de una comunidad restringida (la de los científicos) y la del ciudadano común (Godin y Gingras 2000). Dichos modelos son difícilmente aplicables a nuestra realidad latinoamericana, ya que se han propuesto para países que tienen medianamente resuelto el aspecto educativo formal en ciencias, que invierten significativamente en ciencia y cuyos ciudadanos están conscientes de que sus impuestos apoyan, entre otras cosas, el desarrollo de proyectos científicos y tecnológicos. Por otro lado, tratan escasa o muy colateralmente el efecto de la educación informal sobre la cultura científica, cuando en nuestros países en ocasiones son la opción más importante para la formación ciudadana.

Lo anterior señala entonces que para países latinoamericanos es necesario medir los esfuerzos de la CPC en un contexto en particular, y de allí tratar de ver su contribución en pro de la cultura científica. Desde luego que no bastará con esta consideración, sino que habrá que añadir la contribución de la escuela formal y la formación ciudadana en materia de ciencia y tecnología, que en conjunto permiten a los individuos apropiarse de la ciencia al hacer de estos campos parte de su vida diaria y dotarlos de los elementos indispensables para su actuar en la participación social y la democracia. Un asunto más a considerar es la generación del gusto, incluso estético, por la ciencia, que algunos expertos manejan como componente de la cultura científica (Sánchez 2010). Por ello es que resulta indispensable revisar cómo y de qué forma se está dando acceso a la ciencia al público general, y posteriormente intentar evaluar en qué medida se cumplen los objetivos propuestos por los esfuerzos realizados de CPC en cada comunidad.

Por tanto, antes que buscar medir la apropiación de la ciencia (Godin y Gingras 2000) en nuestros contextos, será necesario determinar en primera instancia la calidad del acercamiento a la ciencia a partir de la CPC a través de los medios de comunicación disponibles, que intentan de entrada sensibilizar a los individuos sobre la presencia de la ciencia en la sociedad. 
De esta manera, será importante registrar en primer lugar si en efecto los medios para la CPC y las acciones derivadas de esta ponen a la ciencia a la vista de los receptores, para que eventualmente les interese, les llegue a atraer y finalmente la logren considerar como una herramienta fundamental de su vida.

De esta manera, la medición más básica del efecto de la CPC sobre la cultura científica sería la detección del esfuerzo que realizan instituciones, sociedades e incluso países, para que la ciencia sea conocida, y así acercarla al ciudadano hasta que este se la apropie como la más convincente manera de explicar el mundo. Es así que podemos decir que en nuestros países es cada vez más indispensable evaluar los esfuerzos realizados en las modalidades que la CPC reviste, en lugar de preocuparnos prematuramente por obtener mediciones de la cultura científica alejadas de nuestro contexto y que por lo mismo no reflejan nuestra realidad.

\section{La evaluación de los alcances de la CPC}

Por las razones anteriores es que a continuación se hace una descripción simplificada de los eventos de CPC en relación con los medios empleados y sus intenciones, así como con la consideración de los receptores, en un intento por presentar un punto de partida previo a la evaluación de la adquisición o de la generación de la cultura científica, en este caso a partir de las variadas modalidades de la CPC.

\section{Los componentes a evaluar en la CPC}

Para tener claro el panorama completo de la comunicación de la ciencia, hay cuatro aspectos a tomar en cuenta: dos corresponden a líneas de índole teórica, y dos son consideraciones de naturaleza práctica. Por el lado teórico, el panorama completo requiere considerar por una parte el efecto buscado por las actividades de comunicación de la ciencia, que forman una línea desde el entretenimiento hasta el aprendizaje informal. Igualmente es necesario contar con una línea que describa la percepción del tipo de público en el que se pretenda incidir, desde masivo hasta individual.

Las consideraciones de tipo práctico se refieren a un tercer aspecto que abarca la diferenciación de los objetivos perseguidos, es decir, a las razones por las que se hace la CPC, y por otro, a las maneras en las que se realiza la CPC y los medios de comunicación más adecuados para lograrla.

En la figura 1 pueden verse los ejes de los efectos y los públicos, estos ejes no hacen referencia a aspectos positivos o negativos de cada uno de ellos, más bien esquematizan los componentes a tomar en cuenta. También se observan los objetivos derivados de sus interacciones, que son el tercer aspecto mencionado; entre tales objetivos se encuentran de la ciencia.

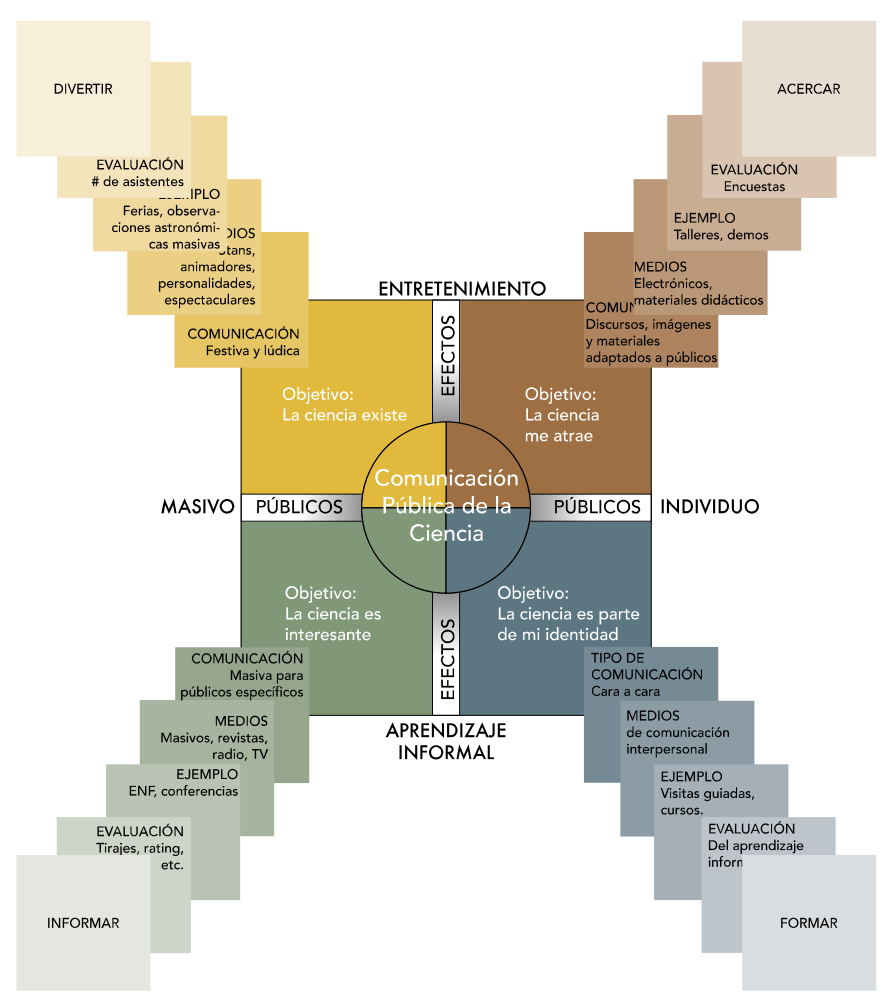

Figura 1. Los componentes para evaluar en la comunicación pública 
dar a conocer la existencia de la ciencia; hacerla ver atractiva, útil e incluso estéticamente valiosa; presentarla de forma interesante, y finalmente, hacer de la ciencia parte de la vida de los individuos. El cuarto aspecto, que depende de los tres anteriores, concierne a las metodologías comunicativas para lograr los objetivos perseguidos con la CPC, entre las que pueden citarse la realización de eventos masivos lúdicos; las presentaciones masivas para públicos específicos; la generación de eventos y materiales para el acercamiento a la ciencia de grupos particulares, hasta la comunicación entre individuos, llamada comunicación de la ciencia cara a cara o bien, comunicación directa de la ciencia.

De aquí se desprende un quinto aspecto que se refiere a los medios idóneos para ejercerla, en su caso, los medios espectaculares como las ferias de ciencia; los medios masivos de comunicación, los ciclos de conferencias o algunos eventos de educación no formal; los materiales creados ex profeso para atraer a diversos públicos hacia la ciencia, como es el caso de los talleres y demostraciones que ocurren en ferias y museos de ciencia, y por último las actividades que permiten la comunicación interpersonal como las visitas guiadas a los espacios de educación informal.

A continuación, se describe el modelo para evaluar cada uno de los componentes de la CPC.

\section{La línea de los efectos}

La cuestión de si la CPC debe buscar entretenimiento o aprendizaje informal ha sido constante (Rennie 2001). Sin embargo, si se considera un continuo entre los dos extremos, cualquier postura en la línea de los efectos determinará, expresa o tácitamente, de qué manera una institución que hace comunicación de la ciencia definirá lo que se comunicará y cómo. Así el efecto dependerá del objetivo planteado en la actividad de CPC.

Aunque las líneas de efectos y de públicos parecieran independientes en primera instancia en realidad están muy conectadas.

\section{La línea de los públicos}

Así como ocurre con los efectos de la CPC, los públicos pueden organizarse en un continuo con dos posiciones contrastantes en los extremos. En un lado están los públicos masivos desconocidos y en la otra los individuos.

Las diferentes visiones de los públicos determinarán el foco de la atención en cada evento de CPC. La consideración de que el público sea masivo requerirá enfocarse en eventos para grupos numerosos y heterogéneos; pero si el foco está en el individuo, entonces se deberán tomar en cuenta las metodologías más adecuadas existentes para hacer llegar el discurso de la CPC a cada persona.

Los dos continuos mencionados de los efectos y los públicos pueden cruzarse para generar cuatro cuadrantes, cada uno de los cuales corresponde a un objetivo particular de la CPC.

\section{Los cuadrantes de la CPC}

\section{E1 cuadrante público masivo-entretenimiento}

En este cuadrante se pueden ubicar las instituciones y promotores de la CPC que se enfocan en los eventos masivos, cuya función es divertir. Por ejemplo, una institución organiza un evento masivo en donde lo que se busca es el entretenimiento de los asistentes, dada la característica, la institución tendrá poca información descriptiva del público, sin embargo, el objetivo que se busca es mostrar que la ciencia existe. Aquí el reto para el comunicador es cómo 
atraer a ese público masivo mediante campañas de promoción, y cómo entretenerlo una vez reunido, utilizando como medios stands, música, presencia de personalidades, etc.

Los eventos que se organizan en este cuadrante buscan la espectacularidad con la intención de poner a la ciencia a la vista de los receptores. En esta modalidad se ubican las ferias de ciencia, observaciones astronómicas masivas, semanas de la ciencia, etc. En la figura 2, se muestra un ejemplo de este cuadrante, en donde se realizaron diferentes actividades para la divulgación de las matemáticas incluyendo la conferencia con un divulgador de renombre (Eduardo Sáenz de Cabezón, en este ejemplo).

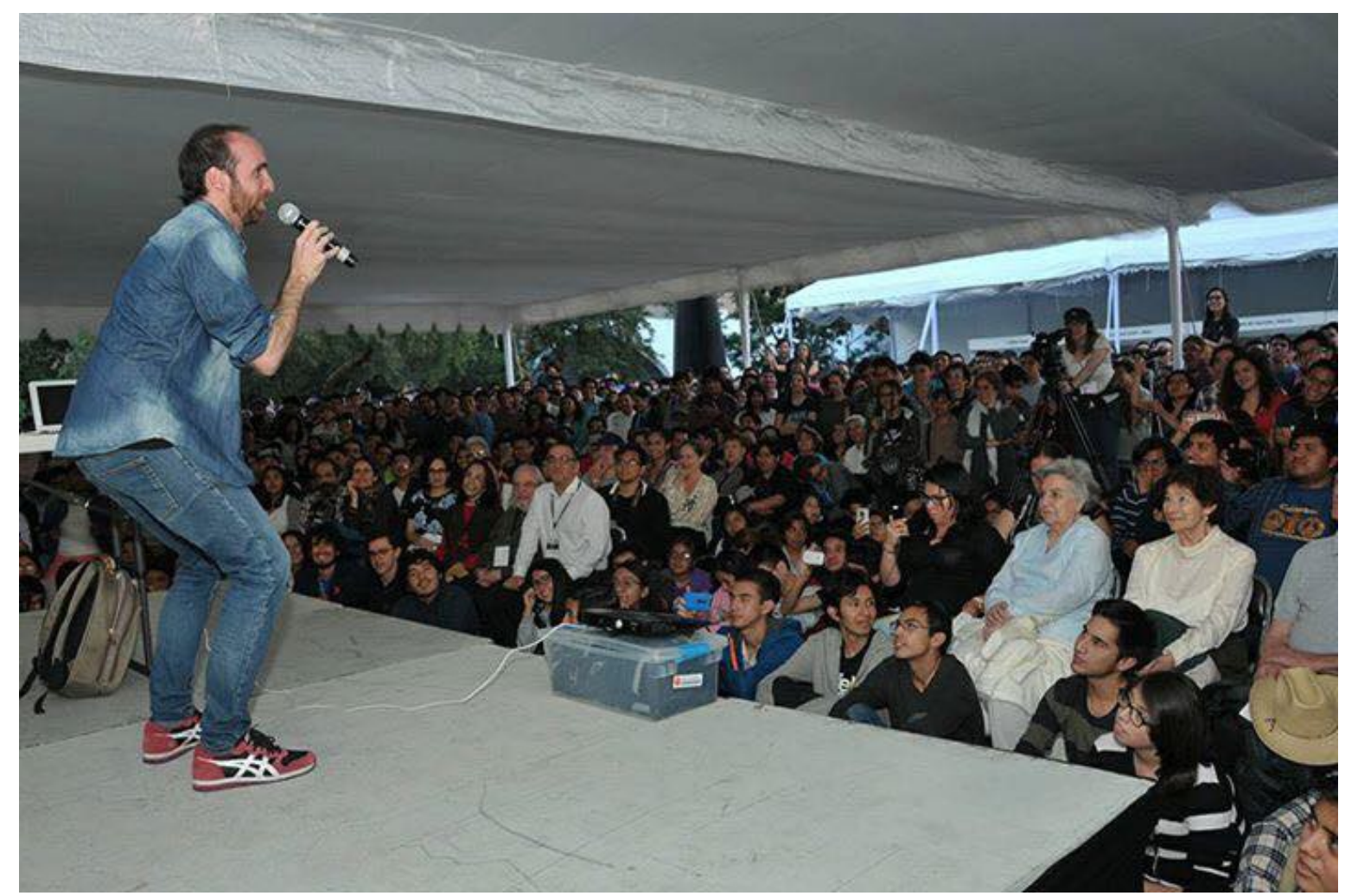

Figura 2. Matemáticas en la calle. Actividades de divulgación del $50^{\circ}$ Congreso Nacional de la Sociedad Matemática Mexicana. Explanada del MUAC del Centro Cultural Universitario, UNAM.

\section{El cuadrante público masivo-aprendizaje informal}

El cuadrante inferior izquierdo representa una posición de la CPC que comparte una visión global del público, pero supone que su participación le hará interesarse por la ciencia y la tecnología. En este cuadrante la comunicación es masiva para públicos específicos. Los eventos de CPC organizados con esta concepción tienen la función de informar, y el objetivo que buscan es que los públicos encuentren que la ciencia es interesante. En este tipo de CPC se encuentran las revistas de divulgación, los documentales científicos, los canales de divulgación de la ciencia en youtube, programas de radio, TV, etc., los cuales tienen en común la periodicidad de sus publicaciones o transmisiones, según sea el caso. En la figura 3, se muestran dos ejemplos de este cuadrante.

\section{El cuadrante individuo-entretenimiento}

El cuadrante superior derecho está enfocado en el individuo sobre el eje de entretenimiento. Aquí las actividades de CPC tienen por objetivo hacer que los participantes se sientan atraídos hacia la ciencia, es decir, que el individuo exprese la ciencia me atrae. Las actividades que caen en este cuadrante son los talleres de ciencia, demostraciones de ciencia, visitas libres a espacios de 

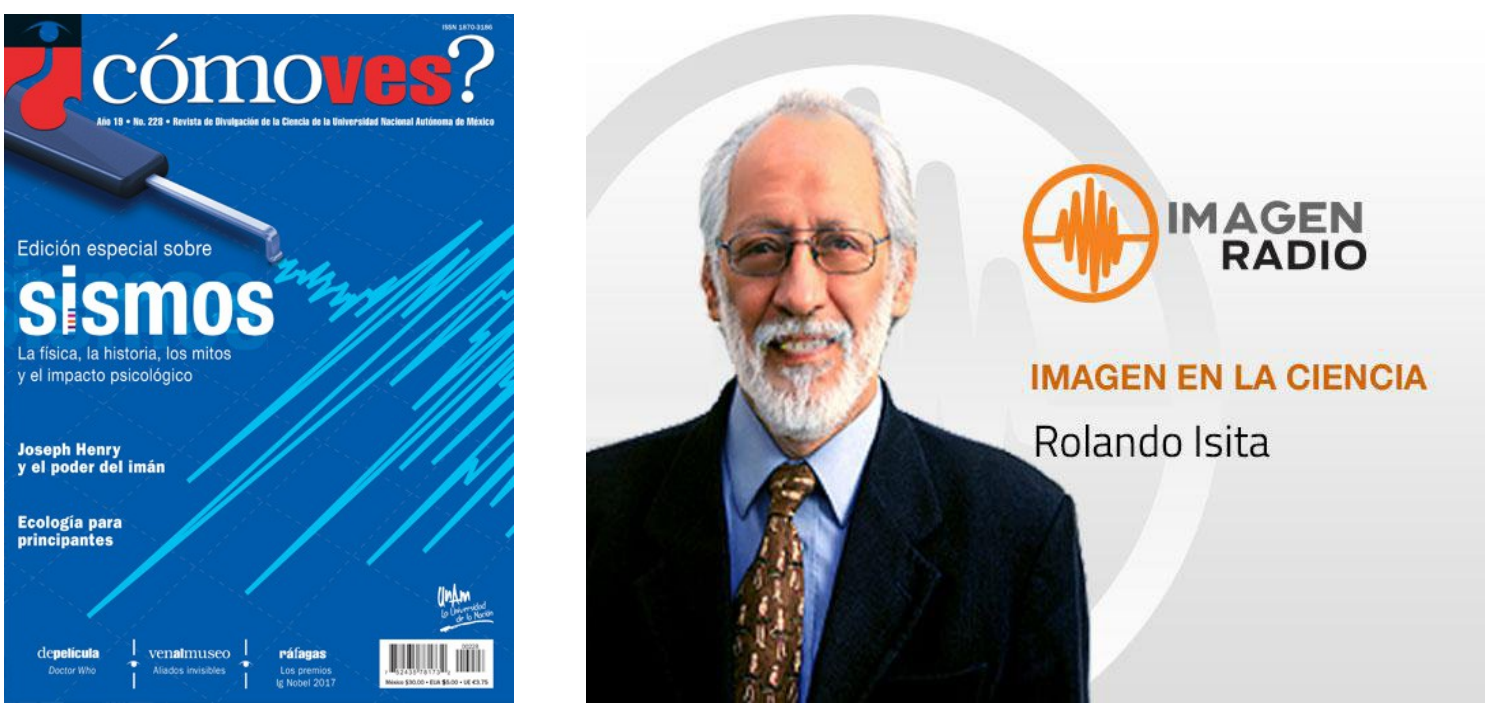

Figura 3. (Izq.) Revista de divulgación de la ciencia ¿Cómo ves? (Der.) Programa de radio Imagen en la Ciencia.

educación informal, etc. Tanto en este cuadrante como en el anterior es donde puede incorporarse con facilidad el gusto estético por la ciencia y el enfoque social de la ciencia. Un ejemplo muy seguido por los comunicadores de la ciencia son los cursos infantiles de verano de ciencia como el que se muestra en la figura 4 y los campamentos científicos para jóvenes.

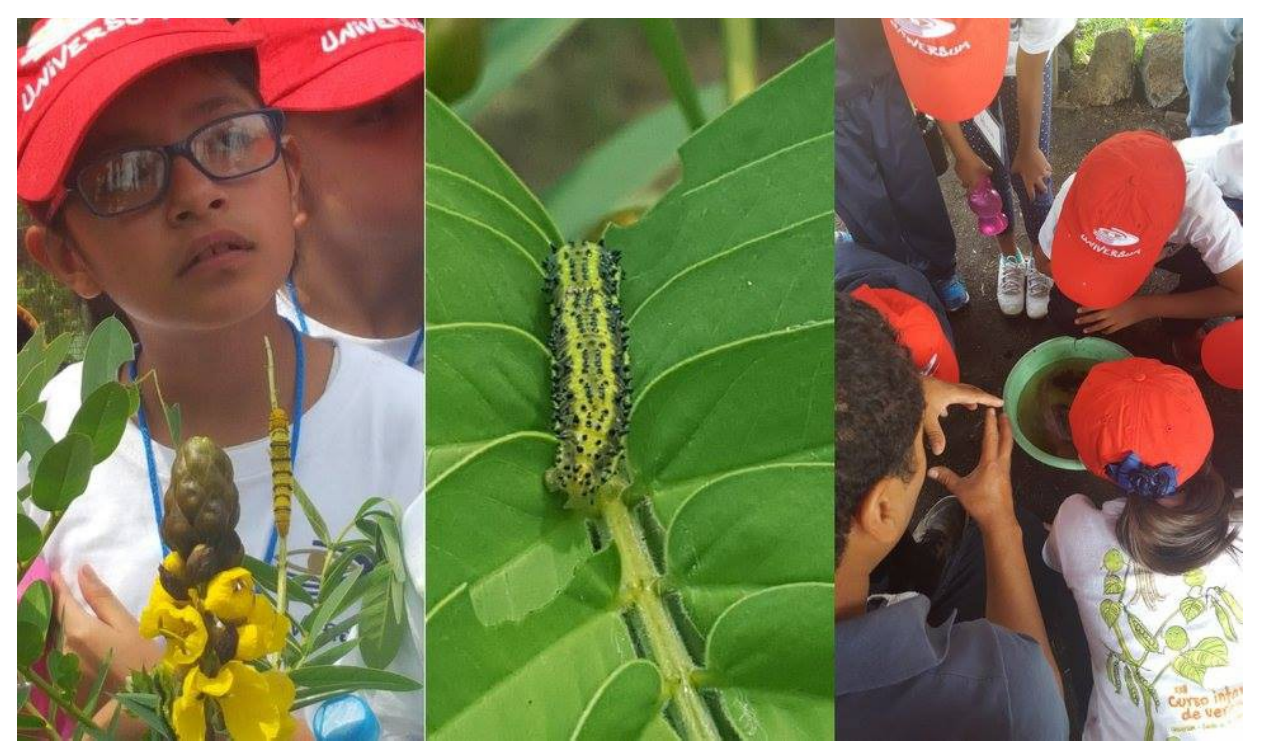

Figura 4. Curso infantil de verano Universum - Casita de las ciencias.

\section{E1 cuadrante individuo-aprendizaje informal}

En este último cuadrante, la CPC es vista como una actividad interpersonal que pretende una formación o una alfabetización científica (Polino, Fazio y Vaccareza 2003); es necesario conocer previamente las características de cada individuo y sus contextos, con objeto de lograr que sienta que la ciencia es parte de su identidad y con énfasis en su formación.

Respecto a los aspectos relacionados con identidad, los resultados de la educación informal en ciencias propuestos por el National Science Council, representan una amplia visión de la manera en que los practicantes y lo expertos caracterizan y miden los efectos de las experiencias de aprendizaje informal. Las seis líneas (strands) que proponen para medirlos 
cubren un amplio rango de enfoques, desde los dirigidos hacia el cambio cognitivo y conceptual, hasta aquellos más relacionados con la participación o la identidad (Rodari 2009).

Un ejemplo de lo que ocurre en este cuadrante son las visitas guiadas a los museos de ciencias donde los mediadores adecuan el discurso a las necesidades individuales de cada visitante. Los eventos que se planean en este cuadrante buscan experiencias que estimulen el aprendizaje informal de los conceptos científicos. En la figura 5, se observa una visita guiada a un simulador esférico del planeta Tierra en el que se muestran diversos fenómenos meteorológicos.

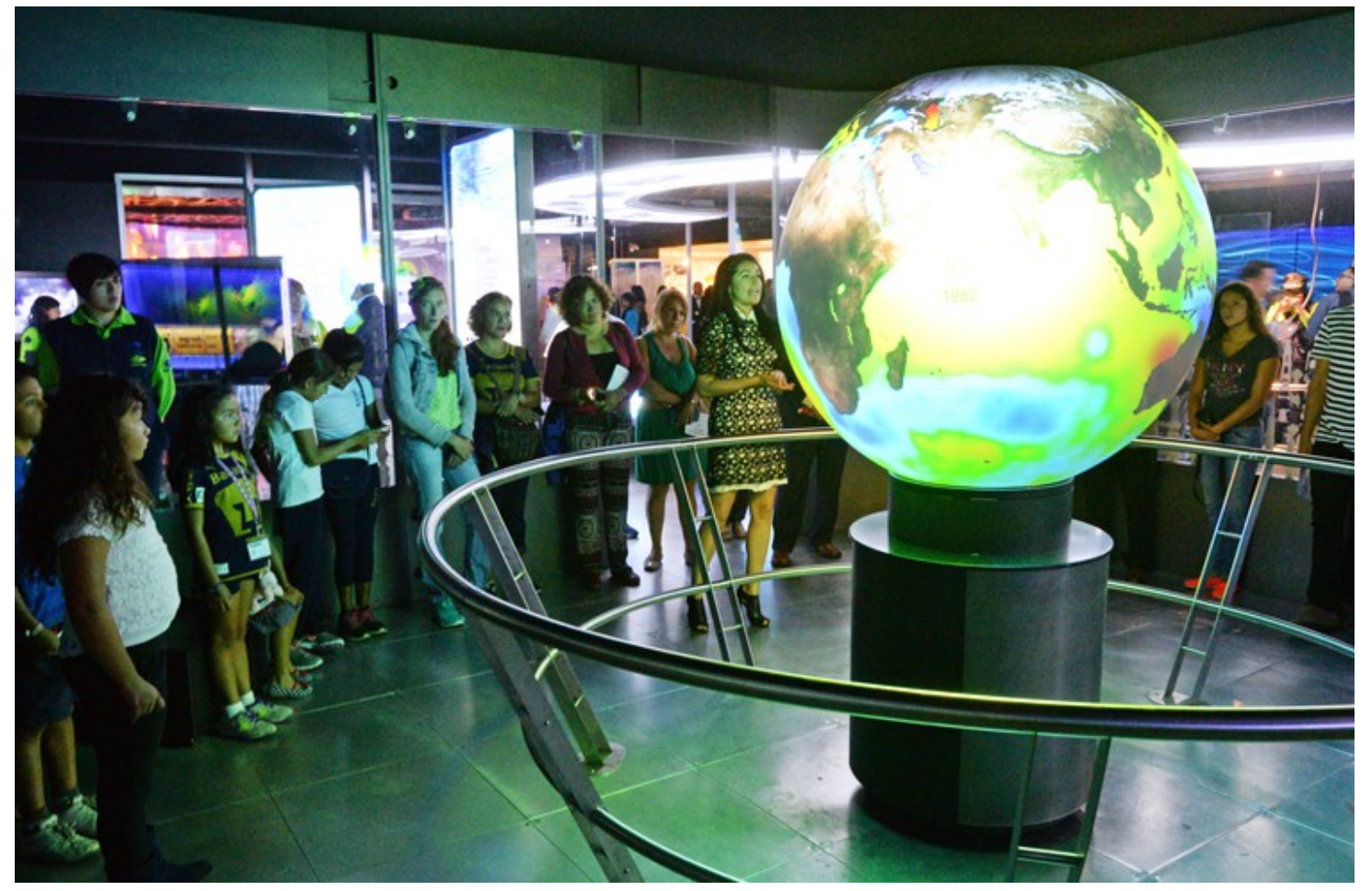

Figura 5. Sala Agua, elemento de la vida, en el Universum Museo de las Ciencias.

\section{Las modalidades de CPC para cada cuadrante}

Una visión de los públicos y de los alcances de la comunicación son esenciales, aunque no suficientes, para plantear las diferentes visiones y las posibilidades de evaluación de las actividades de la CPC. Por tanto, será necesario contar con una imagen completa de cómo llevar a cabo esta comunicación de acuerdo con lo que propone cada cuadrante.

\section{La comunicación de la ciencia y su evaluación para el cuadrante público masivo- entretenimiento}

El reto en este cuadrante en términos de comunicación de la ciencia consiste en cómo estructurar los eventos masivos de CPC para que sean llamativos y diviertan. La forma de evaluarlo será a partir de la consideración del número de eventos efectuados en un cierto periodo y de la concurrencia a estos. Por lo tanto, los resultados esperados en este cuadrante será la cantidad de participantes, mientras más sean se vincula con el éxito del evento masivo.

La comunicación de la ciencia y su evaluación para el cuadrante público masivoaprendizaje informal

En este caso será necesario diseñar los eventos de CPC para audiencias específicas y con objetivos de aprendizaje informal. Para lograr estos objetivos se hará uso principalmente de 
los medios masivos de comunicación y la evaluación considerará la penetración de los medios (tirajes, ratings, etc.). En este caso se incluyen también los ciclos de conferencias, las demostraciones y algunos eventos de educación no formal, cuya evaluación está relacionada con cuestionarios de opinión.

\section{La comunicación de la ciencia y su evaluación para el cuadrante individuo- entretenimiento}

Aquí se utilizan discursos y actividades adaptadas a públicos meta (escritos, audios, materiales didácticos, etc.). Los talleres de ciencia son uno de los ejemplos más conocidos de esta modalidad junto con las demostraciones. La evaluación se realizará mediante encuestas de satisfacción y de sugerencias.

\section{La comunicación de la ciencia y su evaluación para el cuadrante individuo- aprendizaje informal}

En este último cuadrante se buscan experiencias que estimulen el aprendizaje informal a través de actividades de comunicación interpersonal de acuerdo a las necesidades de cada persona, para que sigan participando de la CPC hasta hacer de la ciencia parte de su identidad. En este caso la evaluación se hará a través de cuestionarios, entrevistas estructuradas, grupos de enfoque, etc.

\section{Conclusiones}

Se ha partido de considerar que la consecución de una cultura científica depende de la intervención de varios procesos, mismos que se resumen en la figura 6 . Aquí puede observarse que sobre la generación de la cultura científica intervienen factores como la escolaridad formal y el enfoque social de las ciencias, además de la CPC en los cuatro cuadrantes descritos anteriormente. Hasta ahora esta contribución a la CPC se había dejado de lado en las definiciones de cultura científica.

El breve resumen aquí expuesto pretende ofrecer una forma de considerar la CPC a partir de una rápida visión de las diferentes categorías de sus objetivos. Para poder plasmarlos en prácticas, se requerirá derivar de ellos diferentes métodos de CPC que sean consistentes con las combinaciones particulares de visiones de los públicos y de los efectos buscados a través del empleo de medios de comunicación específicos. Cabe aclarar que un mismo medio puede, con diferente enfoque, ser utilizado en más de Figura 6. Efectos de la comunicación pública de la un cuadrante.

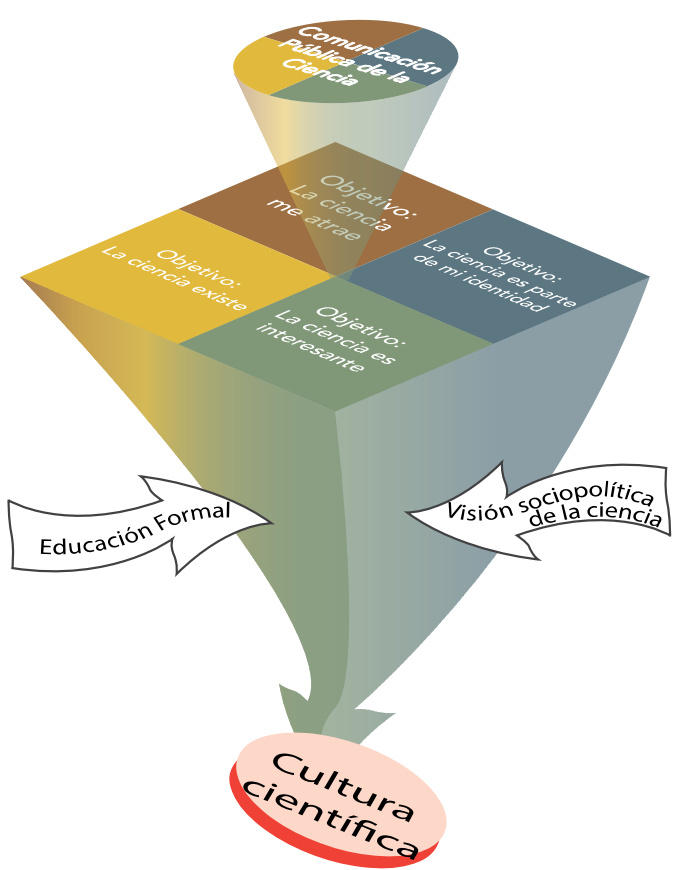

ciencia sobre la cultura científica.

Se ha enfatizado el contraste que existe entre los enfoques meramente masivos y entretenidos, y aquellos donde lo que se busca es un aprendizaje y una identidad.

Las diferencias teóricas provienen de las diferentes maneras de considerar cómo responden los receptores a los esfuerzos de la CPC. Pero cómo se defina la CPC dependerá en todo caso 
de la actividad ejercida por los divulgadores o por los comunicadores de la ciencia, puesto que, finalmente, la manera en que se estudien, investiguen, evalúen o consideren las metas de la CPC será dependiente de la posición que se asuma en los cuadrantes.

Cualquier institución, empresa o grupo que se dedique a la CPC y que realmente busque influir en la cultura científica deberá intentar cubrir los cuatro cuadrantes en lo que a la CPC se refiere. Esta propuesta permite además hacer una revisión del balance de los enfoques de la CPC que se lleve a cabo en distintos ámbitos.

Se ha hecho hincapié en que la formación de una cultura científica también deberá incluir aspectos de los estudios sociales acerca de la ciencia, donde se aporte a los ciudadanos herramientas de discusión y de participación democrática en asuntos sobre ciencia y tecnología. Cabe aclarar que estos últimos puntos podrían ser también producto de la CPC ejercida a través de los cuatro cuadrantes.

\section{Bibliografía}

Bauer M. W., Petkova K., Boyadjieva P. (2000) Public knowledge of and attitudes to science: Alternative measures that may end the "science war". Science, Technology \& Human Values 25 (1), 30-51.

Borun M., Massey C., Lutter T. (1993) Naive knowledge and the design of science museum exhibits. Curator 36 (3), 201-219.

Brown B. A., Reveles J. M. K., Gregory J. (2005) Scientific literacy and discursive identity: a theoretical framework for understanding science learning. Science Education 89, 779-802.

Burns T. W., O'Connor D. J., Stocklmayer S. M. (2003) Science communication: a contemporary definition. Public Understanding of Science 12, 183-202.

Diamond J., Luke J. J., Utall D.H. (2009) Practical Evaluation Guide. Tool for museums and other informal educational settings, 2nd ed. New York, Altamira Press.

Durant J., Evans G., Thomas G. (1995) The relationship between knowledge and attitudes in the public understanding of science in Britain. Public Understanding of Science 9 (4), 57-74.

Falk J., Dierking L. (2012) Lifelong Science learning for adults: the role of freechoice experiences. pp. 1063-1079 en B. J. Fraser et al. (eds.), Second International Handbook of Science Education. New York, Springer International Handbooks of Education.

Gregory J., Miller S. (1998) Science in Museums. Science in Public: Communication, culture and credibility. New York, Plenum Press.

Godin B., Gingras Y. (2000) What is scientific and technological culture and how is it measured? Public Understanding of Science 9 (1), 43-58.

Hooper G. (2008) Generic Learning Outcomes GLO's. Museums, Libraries and Archives Council.

Jenkins E.W. (1994) Public understanding of science and education for action. Journal of Curriculum Studies 26 (6), 601-611.

Lewenstein B. V. (2003) Models of public communication of science and technology. Public Understanding of Science. Cornell University.

Miller J. D. (2001) The acquisition and retention of scientific information by American adults. pp. 93-114 en Free Choice Science Education: how we learn science outside school, J. H. Falk (ed.). New York, Teachers College Press. 
National Research Council (2009) Learning Science in Informal Environments: People, Places and Pursuits. Committee on Learning Science in Informal Environments. L. Philip, L. Bruce, W. S. Andrew, A. F. Michael (eds.). Board on Science Education, Center for Education. Division of Behavioral and Social Sciences and Education. Washington, D.C., The National Academic Press.

Polino C., Fazio M. E., Vaccarezza L. (2003) Medir la percepción pública de la ciencia en los países iberoamericanos. Aproximación a problemas conceptuales. Revista Iberoamericana de Ciencia, Tecnología, Sociedad e Innovación 5, enero-abril.

Rennie L. J. (2001) Communicating science through interactive science centres: a research perspective. pp. 107-121 en Science Communication in Theory and Practice, S. M. Stocklmayer, M. M. Gore, C. Bryant (eds.). Boston, Science and Technology Library, Kluwer Academic Publishers.

Rennie L. J., McClafferty T. P. (1996) Science Centres and Science Learning. Studies in Science Education 27, 53-98.

Reynoso H. E. (2001) La evaluación de la divulgación de la ciencia. Ponencia presentada en la mesa redonda del mismo nombre durante el X Congreso Nacional de Divulgación de la Ciencia. Toluca, Estado de México, México.

Rodari P. (2009) Learning science in informal environments: people, places and pursuits. A review by the US National Science Council (Review). Journal of Science Communication 8 (3), September.

Sánchez A. (2010) La divulgación de la ciencia como literatura. Dirección General de Divulgación de la Ciencia (DGDC). México, Universidad Nacional Autónoma de México (UNAM).

Schauble L., Leinhardt G., Martin, L. A. (1997) Framework for organizing a cumulative research agenda in informal learning contexts. Journal of Museum Education 22 (2 y 3), 311.

Zamarrón G. (2006) De cultura científica y anexas. pp. 129-145 en VVAA, Universidad, comunicación y ciencia: contrastes. México, Universidad de Baja California y Mario Porrúa Editores. 